\title{
Further Comments on the Computation of Modified Bessel Function Ratios
}

\author{
By Marietta J. Tretter and G. W. Walster
}

\begin{abstract}
A theorem by W. J. Thron is used to enhance and further substantiate empirical results by Gautschi and Slavik on the convergence of continued fractions used to compute ratios of modified Bessel functions.
\end{abstract}

1. Thron's Theorem Applied to Continued Fractions for Ratios of Modified Bessel Functions. A theorem by Thron* [2] offers analytic confirmation of recent empirical results [1] on the convergence of continued fractions, cf's, for computing ratios of modified Bessel functions. Defining

$$
K\left(a_{n} / 1\right)=\frac{a_{1}}{1+} \frac{a_{2}}{1+} \frac{a_{3}}{1+} \ldots,
$$

Thron's Theorem 6.1 states:

The continued fraction $K\left(a_{n} / 1\right)$ converges to a value $u$ which satisfies $R(u)$ $\geqslant-1 / 2$ provided that $I^{2}\left(a_{n}\right) \leqslant R\left(a_{n}\right)+1 / 4$ and $\left|a_{n}\right|<M$ for all $n \geqslant 1$. Here $M$ is an arbitrary large positive quantity.

As a consequence of Thron's theorem we can obtain a priori bounds on the truncation error, $2 R_{n}$, of Gauss's and Perron's cf's for modified Bessel function ratios. In the case of Thron's Theorem 6.1, $R_{n}$ is the radius of the value region (nested circles within which the value of the cf lies) of the cf's. From Thron's theorem we thus have

$$
R_{n} \leqslant 1 / \prod_{k=1}^{n}(1+1 /(4 M k)) .
$$

Relatively larger values of $M$ are associated with overall slower convergence.

Both Gauss's and Perron's cf's for $I_{v}(x) / I_{v-1}(x)$ are in the form required by the theorem [1]. For positive $x$ the assumptions of Thron's theorem are trivially satisfied for Gauss's cf and are easily verified for Perron's cf if $v \geqslant 1$. Thus, to apply the theorem, note that when $x>v$, the $a_{n}$ of Perron's cf will be dominated by $1 / a_{0}(x)=$ $x /(x+2 v)$ and the $a_{n}$ of Gauss's cf will be dominated by $a_{1}(x)=(1 / 4) x^{2} / v(v+1)$, which we will refer to as $M_{P}$ and $M_{G}$, respectively. Clearly, as $x \gg v, M_{P}<1$ and $M_{G} \gg 1$. In this case, $R_{n}$, or $2 R_{n}$ (the truncation error), of Gauss's cf is much larger than Perron's, indicating slower convergence of the Gauss cf. These results are given in Table 1.1 which reproduces Table 3.2 of [1] supplemented by values of $M_{P}$ and $M_{G}$

Received March 20, 1978; revised September 5, 1979.

1980 Mathematics Subject Classification. Primary 33A40; Secondary 40A15, 65D1 5.

* Thron's theorem referred to here is actually a special case of an earlier result due to Scott and Wall (Trans. Amer. Math. Soc., v. 47, 1940, pp. 155-172). However, the results of this paper depend on the proof as given by Thron. 
( $k$ in Table 1.1 represents the number of terms required in the Gauss and Perron cf's to obtain relative accuracy of $1 / 210^{-8}$, as originally given in Table 3.2 of [1]). One interesting observation in Table 1.1 is the fact that for $x \gg v, M_{P}$ is increasing slightly as $x$ increases, even though the table indicates, for 8 decimal digit accuracy, that Perron's $\mathrm{cf}$ is converging faster with increasing $x$. This phenomenon is explained by looking at Figure 3.3 in [1]. The increase in $M_{P}$ reflects the fact that, although initial convergence is fast, at extreme accuracies the convergence slows down before regaining speed. The phenomenon of slowing down also becomes more pronounced as $x$ increases. Thus, one must be careful to interpret $M$ as an overall measure of convergence.

TABLE 1.1

Number of terms, $k$, required in the Gauss and Perron cf's to obtain relative accuracy of $1210^{-8}$ (as given in Table 3.2 of [1]), and $M$ as defined above.

\begin{tabular}{|c|c|c|c|c|c|c|c|c|c|c|c|c|}
\hline & \multicolumn{2}{|c|}{10} & \multicolumn{2}{|c|}{20} & \multicolumn{2}{|c|}{40} & \multicolumn{2}{|c|}{60} & \multicolumn{2}{|c|}{80} & \multicolumn{2}{|c|}{100} \\
\hline$x$ & G & $P$ & G & $P$ & G & $P$ & G & $P$ & G & $P$ & G & $P$ \\
\hline $10 \begin{array}{l}\mathrm{k} \\
\mathrm{M}\end{array}$ & $\begin{array}{l}9 \\
.5\end{array}$ & $\begin{array}{r}13 \\
.33\end{array}$ & $\begin{array}{c}7 \\
.25\end{array}$ & $\begin{array}{r}11 \\
.20\end{array}$ & $\begin{array}{l}5 \\
.13\end{array}$ & $\begin{array}{c}9 \\
.11\end{array}$ & $\begin{array}{l}4 \\
.08\end{array}$ & $\begin{array}{l}8 \\
.08\end{array}$ & $\begin{array}{c}4 \\
.06\end{array}$ & $\begin{array}{l}7 \\
.06\end{array}$ & $\begin{array}{c}4 \\
.05\end{array}$ & $\begin{array}{l}7 \\
.05\end{array}$ \\
\hline $20 \begin{array}{l}\mathrm{k} \\
\mathrm{M}\end{array}$ & $\begin{array}{l}14 \\
1\end{array}$ & $\begin{array}{l}13 \\
.5\end{array}$ & $\begin{array}{l}10 \\
.5\end{array}$ & $\begin{array}{r}12 \\
.33\end{array}$ & $\begin{array}{c}7 \\
.25\end{array}$ & $\begin{array}{l}11 \\
.2\end{array}$ & $\begin{array}{l}6 \\
.17\end{array}$ & $\begin{array}{c}9 \\
.14\end{array}$ & $\begin{array}{l}5 \\
.13\end{array}$ & $\begin{array}{c}9 \\
.11\end{array}$ & $\begin{array}{r}5 \\
.1\end{array}$ & $\begin{array}{c}8 \\
.09\end{array}$ \\
\hline $40 \begin{array}{c}\mathrm{k} \\
\mathrm{M}\end{array}$ & $\begin{array}{c}21 \\
3.64\end{array}$ & $\begin{array}{r}10 \\
.66\end{array}$ & $\begin{array}{l}16 \\
1\end{array}$ & $\begin{array}{l}12 \\
.5\end{array}$ & $\begin{array}{l}11 \\
.5\end{array}$ & $\begin{array}{r}12 \\
.33\end{array}$ & $\begin{array}{l}8 \\
.33\end{array}$ & $\begin{array}{r}11 \\
.25\end{array}$ & $\begin{array}{l}7 \\
.25\end{array}$ & $\begin{array}{l}11 \\
.2\end{array}$ & $\begin{array}{r}6 \\
.2\end{array}$ & $\begin{array}{r}10 \\
.17\end{array}$ \\
\hline $60 \begin{array}{l}\mathrm{k} \\
\mathrm{M}\end{array}$ & $\begin{array}{c}27 \\
8.18\end{array}$ & $\begin{array}{l}9 \\
.75\end{array}$ & $\begin{array}{c}21 \\
2.14\end{array}$ & $\begin{array}{l}10 \\
.6\end{array}$ & $\begin{array}{r}14 \\
.75\end{array}$ & $\begin{array}{r}12 \\
.43\end{array}$ & $\begin{array}{l}11 \\
.5\end{array}$ & $\begin{array}{r}12 \\
.33\end{array}$ & $\begin{array}{l}9 \\
.375\end{array}$ & $\begin{array}{r}11 \\
.28\end{array}$ & $\begin{array}{r}8 \\
.3\end{array}$ & $\begin{array}{r}11 \\
.23\end{array}$ \\
\hline $80 \begin{array}{l}k \\
M\end{array}$ & $\begin{array}{c}32 \\
14.55\end{array}$ & $\begin{array}{r}8 \\
.8\end{array}$ & $\begin{array}{c}26 \\
3.81\end{array}$ & $\begin{array}{r}10 \\
.67\end{array}$ & $\begin{array}{l}18 \\
1\end{array}$ & $\begin{array}{l}11 \\
.5\end{array}$ & $\begin{array}{r}13 \\
.67\end{array}$ & $\begin{array}{l}12 \\
.4\end{array}$ & $\begin{array}{l}11 \\
.5\end{array}$ & $\begin{array}{r}12 \\
.33\end{array}$ & $\begin{array}{r}9 \\
.4\end{array}$ & $\begin{array}{r}11 \\
.29\end{array}$ \\
\hline $100_{M}^{k}$ & $\begin{array}{c}37 \\
22.72\end{array}$ & $\begin{array}{c}7 \\
.83\end{array}$ & $\begin{array}{c}30 \\
5.95\end{array}$ & $\begin{array}{l}9 \\
.71\end{array}$ & $\begin{array}{r}21 \\
1.52\end{array}$ & $\begin{array}{r}11 \\
.56\end{array}$ & $\begin{array}{r}16 \\
.83\end{array}$ & $\begin{array}{r}11 \\
.45\end{array}$ & $\begin{array}{c}13 \\
.625\end{array}$ & $\begin{array}{r}12 \\
.38\end{array}$ & $\begin{array}{l}11 \\
.5\end{array}$ & $\begin{array}{r}12 \\
.33\end{array}$ \\
\hline
\end{tabular}

\begin{tabular}{|c|c|c|c|c|c|}
\hline \multicolumn{2}{|l|}{$x$} & \multicolumn{2}{|c|}{$\nu=1$} & \multicolumn{2}{|c|}{$y=x$} \\
\hline & & G & p & $G$ & $P$ \\
\hline 100 & $\begin{array}{l}k \\
M\end{array}$ & $\begin{array}{l}45 \\
1250\end{array}$ & $\begin{array}{l}5 \\
.980\end{array}$ & $\begin{array}{l}11 \\
.5\end{array}$ & $\begin{array}{l}12 \\
.33\end{array}$ \\
\hline 200 & $\begin{array}{l}k \\
M\end{array}$ & $\begin{array}{l}63 \\
5000\end{array}$ & $\begin{array}{l}4 \\
.990\end{array}$ & $\begin{array}{l}11 \\
.5\end{array}$ & $\begin{array}{l}11 \\
.33\end{array}$ \\
\hline 500 & $\begin{array}{l}k \\
M\end{array}$ & $\begin{array}{l}100 \\
31250\end{array}$ & $\begin{array}{l}4 \\
.996\end{array}$ & $\begin{array}{l}11 \\
.5\end{array}$ & $\begin{array}{l}11 \\
.33\end{array}$ \\
\hline 1000 & $\begin{array}{l}k \\
M\end{array}$ & $\begin{array}{l}142 \\
1.25 \times 10^{5}\end{array}$ & $\begin{array}{l}3 \\
.998\end{array}$ & $\begin{array}{l}11 \\
.5\end{array}$ & $\begin{array}{l}11 \\
.33\end{array}$ \\
\hline 10000 & $\begin{array}{l}k \\
M\end{array}$ & $\begin{array}{l}452 \\
1.25 \times 10^{7}\end{array}$ & $\stackrel{3}{1.000}$ & $\begin{array}{l}12 \\
.5\end{array}$ & $\begin{array}{l}11 \\
.33\end{array}$ \\
\hline
\end{tabular}


Although not as precise as thorough empirical results, Thron's theorem is quite useful in exposing dramatic and overall convergence properties of appropriate cf's with a minimum of effort.

\section{0-Q BAB}

Pennsylvania State University

University Park, Pennsylvania 16802

\section{Control Data Corporation}

HQN060

P. O. Box 0

Minneapolis, Minnesota 55440

1. W. GAUTSCHI \& J. SLAVIK, "On the computation of modified Bessel function ratios," Math. Comp., v. 32, 1978, pp. 865-875.

2. W. J. THRON, "Convergence regions for continued fractions and other infinite processes," Amer. Math. Monthly, v. 68, 1961, pp. 734-750. 